\title{
Monitoring and Managing 4G Network Elements Using Rich UI Frameworks
}

\author{
Sree Lakshmi Addepalli ${ }^{1}$, Sree Gowri Addepalli ${ }^{2}$ \\ Dept., of Computer Engineering, Vivekanand Education Society's Institute Of Technology, Chembur, Mumbai, India ${ }^{1,2}$
}

\begin{abstract}
Network management systems provides an integrated system for sharing device information across management applications, automation of device management tasks, visibility into the health and capability of the network, and identification and localization of network trouble. By using common centralized systems and networkinventory knowledge, a Network management system delivers a unique platform of cross-functional management capabilities that reduces network administration overhead. An element management system (EMS) consists of systems and applications for managing network elements (NE) on the network element-management layer (NEL) of the Telecommunications (TMN) model. The element management system's key functionality is divided into five key areas - fault, configuration, accounting, performance and security (FCAPS). Portions of each of the FCAPS functionality fit into the TMN models. This paper presents a service delivery platform which aims to develop an enterprise web application that manages these $4 \mathrm{G}$ network elements through various modules to meet the required Quality of Service (QoS) using RichFaces framework. RichFaces is an open source Ajax enabled component library for JavaServer Faces which allows easy integration of Ajax capabilities into enterprise application development.
\end{abstract}

General Terms

SDP - Service Delivery Platform

RAM - Resource Assurance Management

KPI - Key Performance Indicator

EMS - Element Management System

NEL - Network Element Management Layer

$\mathrm{NE}$ - Network Elements

TMN - Telecommunications model

FCAPS - Fault, Configuration, Accounting, Performance and Security

Keywords

Element management system (EMS), FCAPS (fault, configuration, accounting performance and security) JavaServer Faces (jsf), RichFaces, JavaEE, JBoss.

\section{INTRODUCTION}

$4 \mathrm{G}$ Fourth generation wireless system is a packet switched An element management system manages one or more of a wireless system with wide area coverage and high specific type of telecommunications network element. throughput. It is designed to be cost effective and to Typically, the EMS manages the functions and capabilities provide high spectral efficiency. The $4 \mathrm{~g}$ wireless uses within each $\mathrm{NE}$ but does not manage the traffic between Orthogonal Frequency Division Multiplexing (OFDM), different NEs in the network.

Ultra Wide Radio Band (UWB), and Millimeter wireless. Data rate of $20 \mathrm{mbps}$ is employed. The high performance is achieved by the use of long term channel prediction, in both time and frequency, scheduling among users and smart antennas combined with adaptive modulation and power control. Frequency band is 2-8 GHz. It gives the ability for worldwide roaming to access cell anywhere. It supports for interactive multimedia, voice, streaming video, Internet, and other broadband services. High speed, high capacity, and low cost per bit Global access, service portability, and scalable mobile services Seamless switching, and a variety of Quality of Service driven services. To provide efficient $4 \mathrm{G}$ services there should be proper communication and management between various nodes or network elements. Element management system To support management of the traffic between itself and other NEs, the EMS communicates upward to higher-level network management systems (NMS) as described in the telecommunications management network layered model.

Examples of elements which can be managed through the EMS interfaces are Cable telephony media gateway, Media gateway, Soft switch, Video compression technology provider, Wireless broadband provider, and Smart electricity meters.

EMS/NMS manages information needs like Performance Counters, Alarms and Configuration Parameters of various nodes. To get this information EMS/NMS have various monitoring tools namely:

(EMS) plays an important role here. 


\section{A. Fault monitoring tool}

In network management, fault management is the set of functions that detect, isolate, and correct malfunctions in a telecommunications network, compensate for environmental changes, and include maintaining and examining error logs, accepting and acting on error detection notifications, tracing and identifying faults, carrying out sequences of diagnostics tests, correcting faults, reporting error conditions, and localizing and tracing faults by examining and manipulating database information.

When a fault or event occurs, a network component will often send a notification to the network operator using a protocol such as simple network management protocol (SNMP). An alarm is a persistent indication of a fault that clears only when the triggering condition has been resolved. A current list of problems occurring on the network component is often kept in the form of an active alarm list such as is defined in RFC 3877, the Alarm management information base (MIB). A list of cleared faults is also maintained by most network management systems.

Fault management systems may use complex filtering systems to assign alarms to severity levels. These can range in severity from debug to emergency, as in the syslog protocol. Alternatively, they could use the ITU X.733 Alarm Reporting Function's perceived severity field. This takes on values of cleared, indeterminate, critical, major, minor or warning. It is considered good practice to send a notification not only when a problem has occurred, but also when it has been resolved. The latter notification would have a severity of clear.

A fault management console allows a network administrator or system operator to monitor events from multiple systems and perform actions based on this information. Ideally, a fault management system should be able to correctly identify events and automatically take action, either launching a program or script to take corrective action, or activating notification software that allows a human to take proper intervention (i.e. send email or SMS text to a mobile phone). Some notification systems also have escalation rules that will notify a chain of individuals based on availability and severity of alarm.

\section{B. KPI or Raw counter monitoring tool}

Key performance indicators define a set of values used to measure against. These raw sets of values, which are fed to systems in charge of summarizing the information, are called indicators. Indicators identifiable and marked as possible candidates for KPIs can be summarized into the following sub-categories:

- Quantitative indicators that can be presented with a number.

- Qualitative indicators that can't be presented as a number.
- Leading indicators that can predict the outcome of a process

- Lagging indicators that present the success or failure post hoc

- Input indicators that measure the amount of resources consumed during the generation of the outcome

- $\quad$ Process indicators that represent the efficiency or the productivity of the process

- Output indicators that reflect the outcome or results of the process activities

- $\quad$ Practical indicators that interface with existing company processes.

- Directional indicators specifying whether or not an organization is getting better.

- Financial indicators used in performance measurement and when looking at an operating index.

A KPI can follow the SMART criteria.

This means the measure has a Specific purpose for the business, it is Measurable to really get a value of the KPI, the defined norms have to be Achievable, the improvement of a KPI has to be Relevant to the success of the organization, and finally it must be Time phased, which means the value or outcomes are shown for a predefined and relevant period.

In order to be evaluated, KPIs are linked to target values, so that the value of the measure can be assessed as meeting expectations or not.

\section{SYSTEM DESIGN}

\section{A. CONCEPT}

The web application is developed to manage and monitor the 4-G network elements using UI Interface and KPI in which we manage and monitor all the nodes which are being used in $4 \mathrm{~g}$ network, to observe that all the nodes are performing well or whether they need any maintenance work.

If any node is not performing well we can get the reason behind that and can resolve it at the same time.

It'll also display the way to resolve that particular error because in future this application will not be under developer's hand, it will be used by the nontechnical person who may not have the enough knowledge about how to resolve those errors.

It'll create the users, assign role to them, manage the alarms and view the raised alarms, configuring the parameters, view the counters in particular module and creates and modify KPI to display the reports.

Nodes are supported By SDP-RAM (Service Delivery Platform - Resource Assurance Management). 


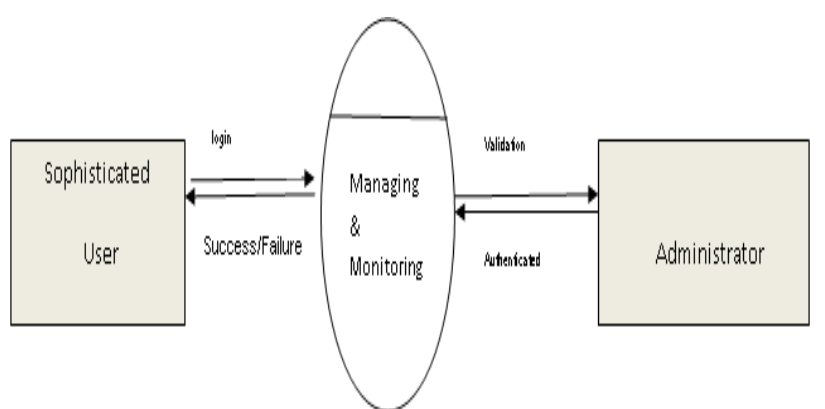

Figure: Context Level Data Flow Diagram

\section{B. IMPLEMENTATION}

The web application is a rich, feature-complete GUI for easy operations and maintenance of the network element nodes. The following modules have been worked upon:

\section{Login Module}

Login Module is a common module as a web based application, where a registered subscriber can login into the SDP-RAM. If user is not registered, an error message is shown in the portal. There is validation over various conditions like login credentials do not match special character validation etc.

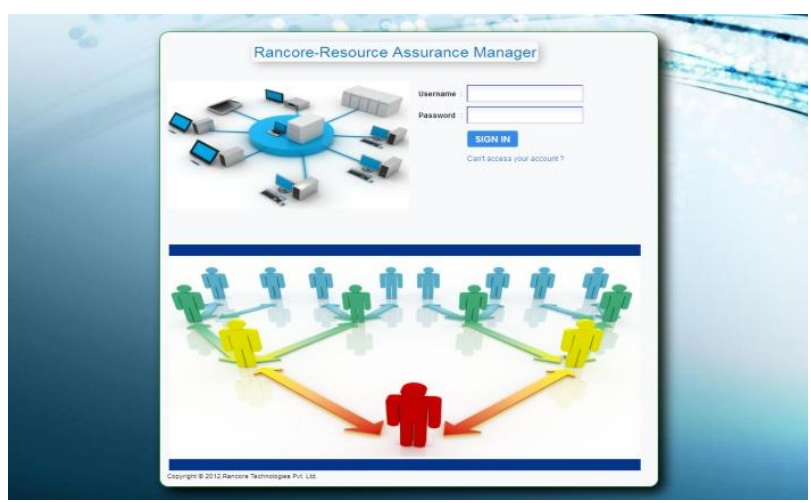

Figure 1: Login Module

\section{User Module}

This module allows the operator to perform user management like creating a new user, modifying a user, viewing details of a user and deleting the user.

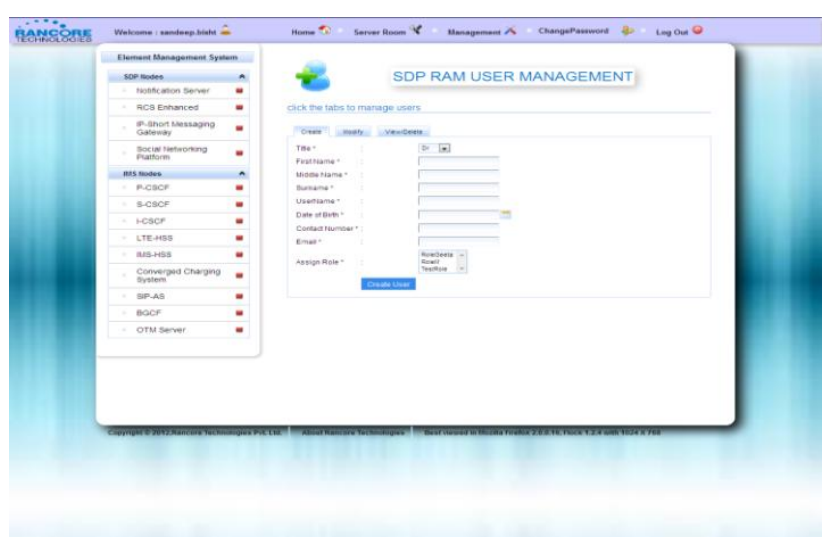

Figure 2: User Module

\section{Role Module}

This module allows the user to create a role, modify, delete and view a particular role of a user or an administrator.

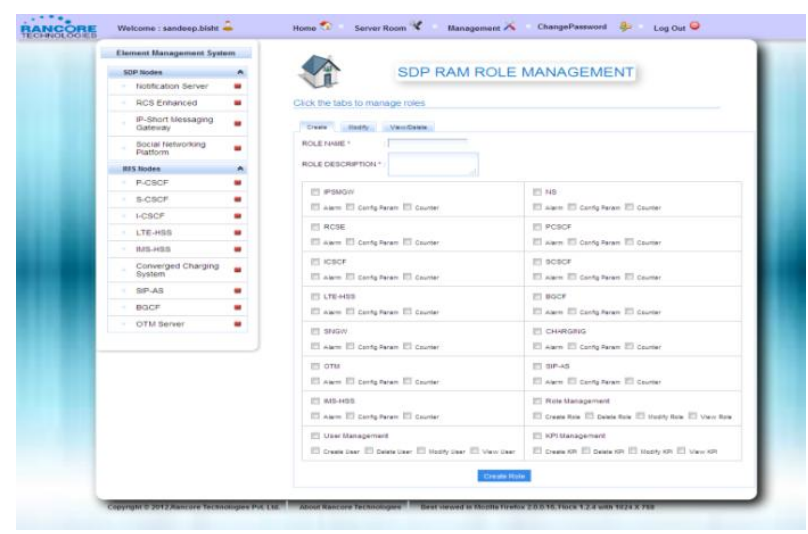

Figure 3: Role Module

\section{Alarm Module}

In this module the operator manages the alarms and views the raised alarms. It also clears all minor alarms and individual alarms.

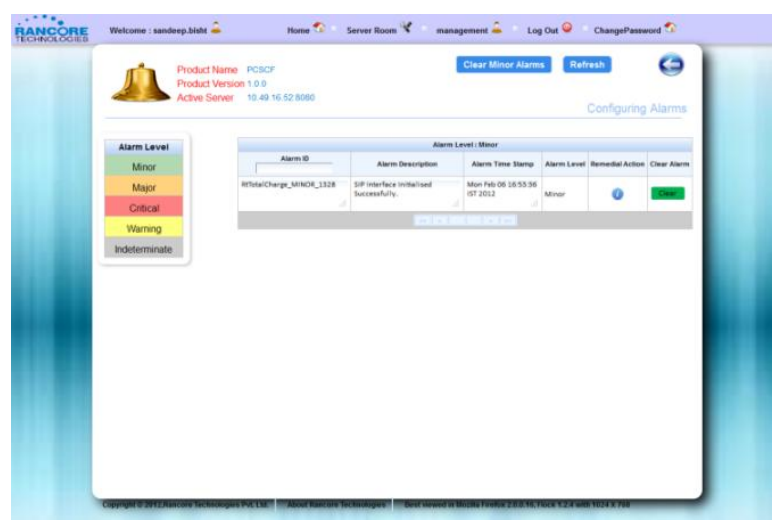

Figure 4: Alarm Module

\section{Counter Module}

This module allows the user to view the counters in particular node and reset the counter.

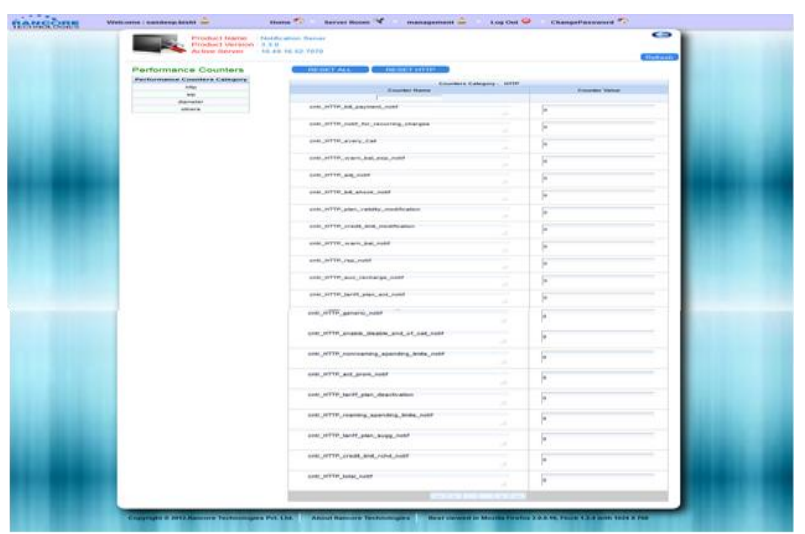

Figure 5: Counter Module 


\section{Configuration Parameter Module}

This module allows the user to view the configuration parameter for particular node. This module sets the scalar parameter and tabular parameter.

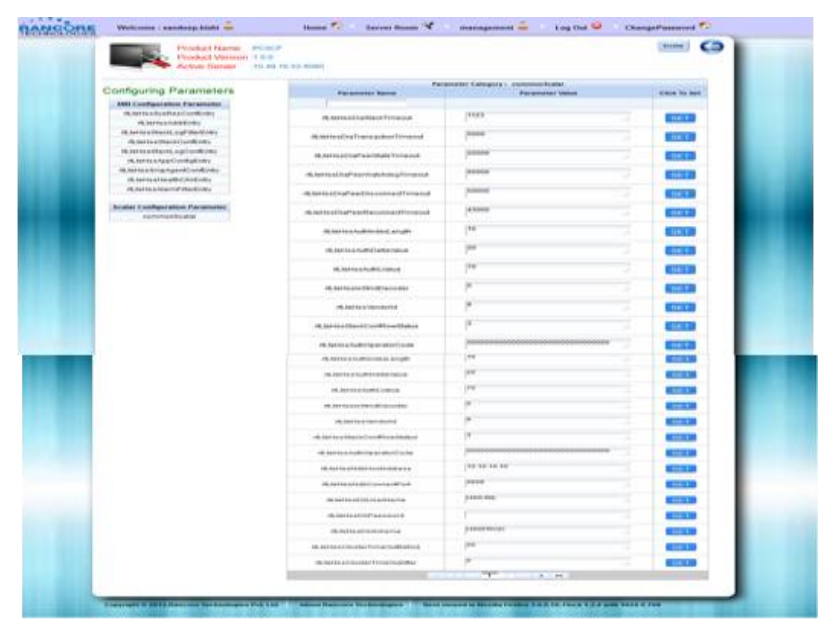

Figure 6: Configuration Parameter Module

\section{KPI Module}

This module allows the user to manage the KPI module by performing CRUD operations (Create, Delete, and Update) on the KPI.

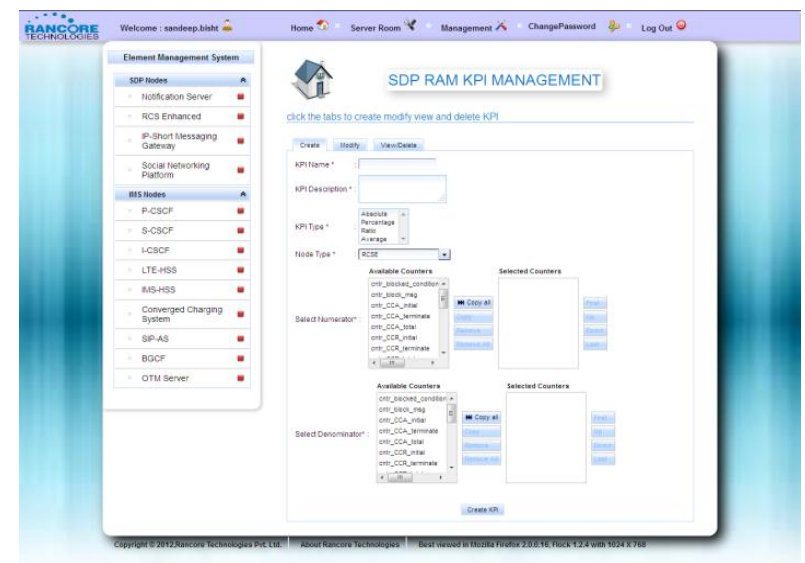

Figure 7: KPI Module

\section{USING SDP-RAM}

The complete SDP infrastructure lies on the underlying JBoss servers. All parts of the SDP viz. customer portal, managed services portal, Telecom services, and instant messaging services are deployed on JBoss Enterprise Application Platform. It contains all the designed modules.

The login page allows the operator login; the user should enter the Username and password in case of invalid username and password appropriate error message will be shown.

The home page allows the operator to view the nodes that are currently up or in existence, the operator can click on an appropriate node icon link in order to perform the respective management related to that node.

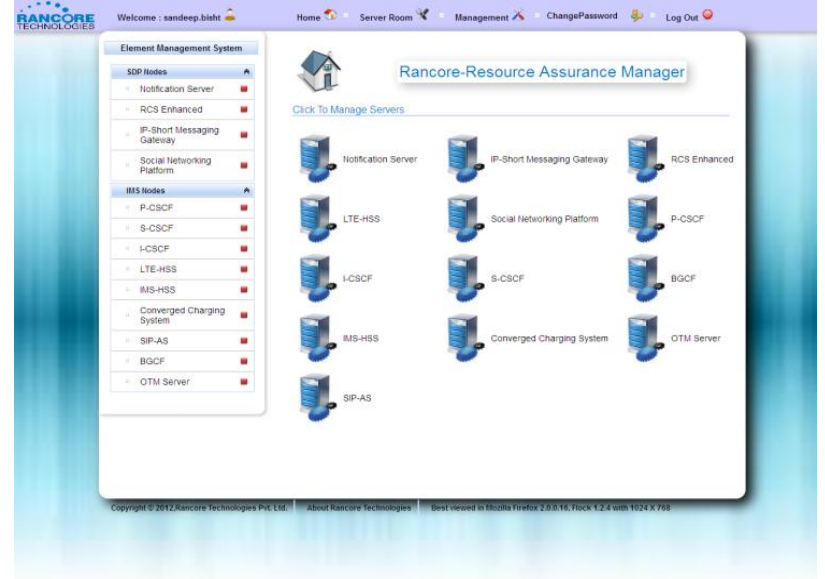

Figure 8: Home Page of SDP-RAM

The navigation bar page will give information about the nodes that are supported by SDP-RAM.

Each node name is accompanied by an icon image beside, if the icon is green color it will indicate that currently the corresponding node is alive, further the icon color will turn red when the corresponding node goes down.

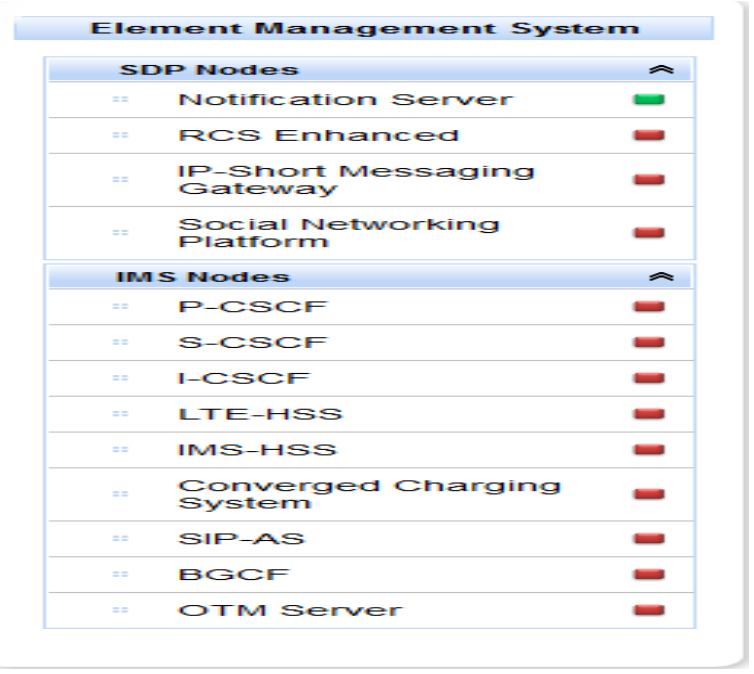

Figure 9: Navigation Page of SDP-RAM

The help page will guide the operator to get information related to server, configuration parameter, performance counter and alarm .

The page includes icons for server help, configuration parameter help, performance counter help and alarm help.

The operator can click on the configuration parameter help link to get an overview about the configuration parameter supported by the node.

The operator can click on the alarm help link to get an overview about the various levels of alarms and the field that it supports.

The operator can click on the performance counter help link to get an overview about the counter supported by the node. 


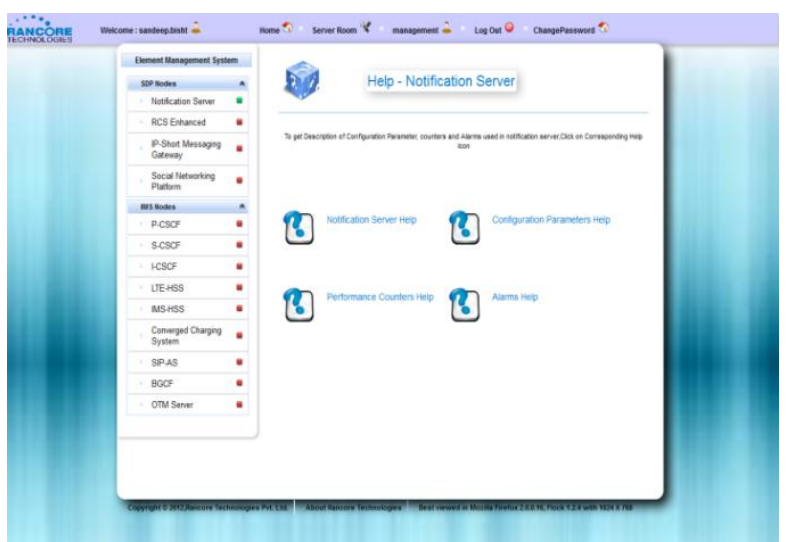

Figure 10: Help Page of SDP-RAM

In the user management module the operator will select the user management option and then select the create tab. The operator is required to enter information like username, first name, surname, email-id and assign a role to the user.

The role management page allows the user to navigate through the different section of the Role Management Module. The operator will select the role management option and then select the create tab. In this module the operator can create an appropriate role that can further be assigned to a particular user to restrict the user privileges.

The dashboard page will indicate the operator the number of alive servers that are in existence for the particular node type. This page will show details of the server such as Sever IP, Sever port, timestamp indicating the time when the server came into existence and the status will indicate whether the server is up or down.

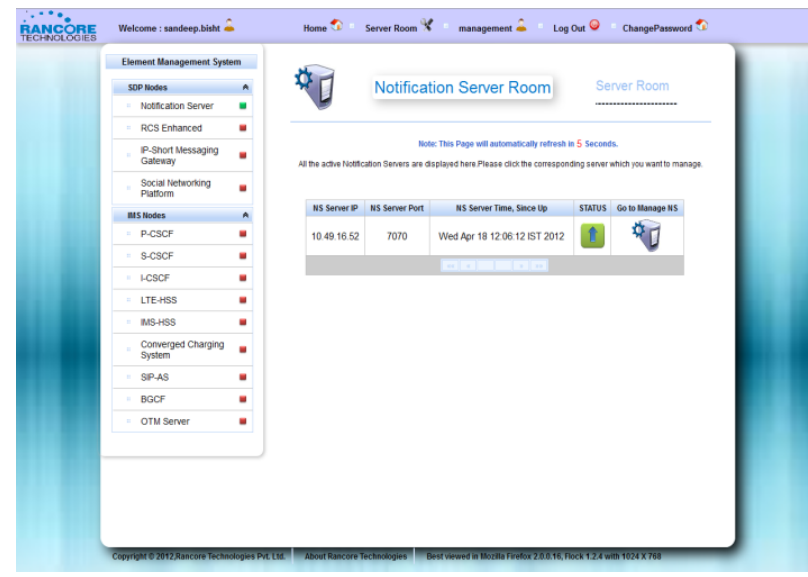

Figure 11: Dashboard Page of SDP-RAM

The manage page shows icons to perform operations related to Configure Parameter management, Alarm management, Counter management for the node

The configure parameter page allows the user to navigate through the different operations of the Parameter Management Module.

Configure

This module allows the operator to view the various configuration parameter related to the particular node.
The alarm page allows the user to navigate through the different operations of the Alarm Management Module. This page allows the operator to view the various alarm levels and the alarms that are raised, this sections gives information about the alarm such as Alarm ID , Alarm description, Alarm timestamp, Alarm level . The operator can clear particular alarm. The operator is also privileged to clear all minor alarms.

The counter page allows the user to navigate through the different operations of the Alarm Management Module. This page allows the operator to view the various alarm levels and the alarms that are raised, this sections gives information about the alarm such as Alarm ID , Alarm description, Alarm timestamp , Alarm level . The operator can clear particular alarm. The operator is also privileged to clear all minor alarms.

\section{SYSTEM ENVIRONMENT}

\section{A. SDP MANAGEMENT INTERFACES}

JBoss Enterprise Application Platform deployed on Red Hat Enterprise Linux plays an extremely critical role in building a state-of-the-art fault tolerant Service Delivery Platform (SDP). It use http, https, and SOAP protocols to achieve web based Operational Goals related to services, subscriber query and order management. SDP supports management from a variety of open interfaces, which can be leveraged for integrating with northbound systems such as Network Management Systems (NMS). The standard open interfaces supported by the SDP are REST (Representational State Transfer) and SOAP (Simple Object Access Protocol).

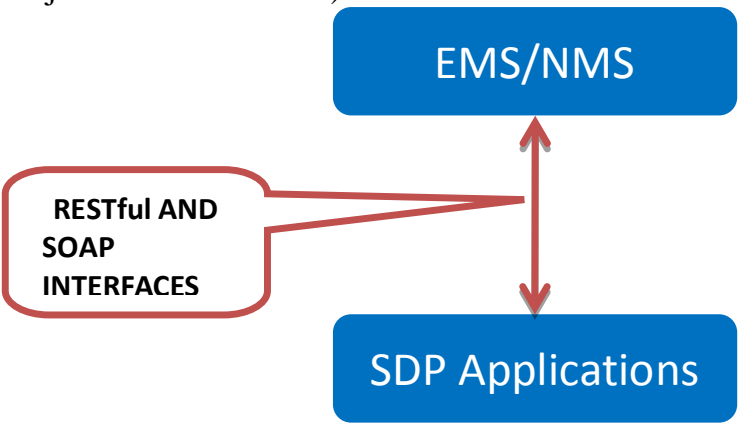

Figure 12: SDP MANAGEMENT INTERFACES

REST is an abbreviation on Representational State Transfer. REST defines a set of architectural principles by which developer can design Web services that utilize system's resources. REST uses HTTP and is stateless. It exposes directory structure-like URIs and transfers XML, JavaScript Object Notation (JSON).

\section{B. TECHNOLOGIES USED 1. Java Enterprise Edition}

The platform provides an API and runtime environment for developing and running enterprise software, including network and web services, and other large-scale, multitiered, scalable, reliable, and secure network applications. The platform incorporates a design based largely on 
modular components running on an application server. A Java EE application server can handle transactions, security, scalability, concurrency and management of the components it is deploying.

\section{Java Server Faces}

Java Server Faces is a standard Java framework for building user interfaces for Web applications. It simplifies the development of the user interface. Without a comprehensive framework designed for enterprise Web application development, these core technologies can often lead to a variety of development and maintenance problems. Java Server Faces avoids problems by offering a robust, "best of breed" framework with well-established development patterns, built upon the experience of many preexisting Java Web development frameworks.

\section{Rich Faces}

Rich Faces is an open source framework hosted by JBoss that adds Ajax capability into existing JSF applications without resorting to JavaScript. Rich Faces leverages Java Server Faces framework including lifecycle, validation, conversion facilities and management of static and dynamic resources. Rich Faces components with built-in Ajax support and a highly customizable look-and-feel can be easily incorporated into JSF applications. Rich Faces is more than just a component library for Java Server Faces. It adds:

- Skinability (easily changes and update application look and feel).

- $\quad$ Component Development Kit (CDK) to assist in constructing Java Server Faces components.

- Dynamic Resource Framework and component based Ajax control components.

\section{RESTful Web Services}

A RESTful web service is a simple web service implemented using HTTP and the principles of REST. It is a collection of resources, with four defined aspects:

- $\quad$ The base URI for the web service.

- The Internet media type of the data supported by the web service is often JSON, XML or YAML.

- The set of operations supported by the web service using HTTP methods (e.g., GET, PUT, POST, or DELETE)

- $\quad$ The API must be hypertext driven.

\section{AJAX}

Asynchronous JavaScript and XML is new web development technique used for the development of most interactive website. Ajax helps in making the web application more interactive by retrieving small amount of data from web server and then showing it on the application without refreshing the page.

\section{Java Server Pages}

JSP is a technology that helps software developers create dynamically generated web pages based on HTML, XML, or other document types. To deploy and run Java Server

Pages, a compatible web server with a servlet container, such as Apache Tomcat is required.

\section{Servlets}

Servlet is a Java programming language class used to extend the capabilities of servers that host applications access via a request-response programming model. Although servlets can respond to any type of request, they are commonly used to extend the applications hosted by Web servers. Thus, it can be thought of as a Java Applet that runs on a server instead of a browser.

\section{DEPLOYMENT}

The application is deployed on JBoss Application Server. The JBoss Enterprise Application Platform is a subscription-based/open-source Java EE-based application server runtime platform used for building, deploying, and hosting highly-transactional Java applications and services. Because it is Java-based, the JBoss application server operates cross-platform: usable on any operating system that supports Java. Its Key features support to Java Server Faces (JSF), including Rich Faces and Web application services, including Apache Tomcat for Java Server Pages (JSP) and Java Servlets.

\section{CONCLUSION}

$4 \mathrm{G}$ wireless networks not only enable more efficient, scalable, and reliable wireless services but also provides wider variety of services. These opportunities come with a need for rethinking our security, privacy, architect and billing technologies that have been used for previous generations. Hence it is very important to manage and monitor the network elements to enable efficient and reliable services.

\section{REFERENCES}

[1] Network Management System by Douglas W. Stevenson

[2] Practical Rich Faces, 2nd Edition, Max Katz, Ilya Shaikovsky

[3] Core Java Server Faces by David Geary (Author), Cay S. Horstmann (Author)

[4] Head First Servlets \& JSP, Second Edition

[5] refcardz.dzone.com/refcardz/richfaces-40-next-generation

[6] RESTful Web Services, Leonard Richardson, Sam Ruby

[7] Rich faces Tutorial,http://www.mastertheboss.com/jbossweb/richfaces/richfaces-4-quick-tutorial

[8] JavaEE,Documentation, http://www.oracle.com/technetwork/java /javaee/documentation/tutorials-137605.html xadel,htt p:// livedem o.exadel.com/richfaces-demo/index.jsp 